\title{
Effective Image Co-Segmentation using Modified Higher Order Algorithm
}

\author{
T. Sandeep Kumar, K. Sreedhar Reddy
}

\begin{abstract}
A young interactive photograph co segmentation algorithm the usage of possibility estimation then greater method electricity optimization is proposed because of extracting frequent foreground objects beside a crew concerning related images. Our approach introduces the greater method clique's, power into the co-segmentation optimization process successfully. $A$ region-based possibility fixity method is preceding celebrated in conformity with provide the formerly skills because our higher kilter strength function. Then, a current co-segmentation electricity characteristic the usage of higher kilter cliques is developed, who may efficaciously co-segment the foreground objects with vast appearance editions beyond a crew about photographs among complicated scenes. Both the quantitative or qualitative pilot results over consultant datasets exhibit that the rigor on our co-segmentation consequences is lots higher than the cutting-edge co-segmentation methods.
\end{abstract}

Keywords: Co-segmentation, Strength, Optimization, Foreground, Background, Scribbles, Likelihood, Smoothing filters.

\section{INTRODUCTION}

Photo co-segmentation is generally referred namely mutually partitioning a associate of pics within the foreground and heritage components. The concept on co-segmentation is advance added with the aid about rother et al. [1] of which she concurrently phase widespread foreground objects out of a yoke on pictures. The co-segmentation trouble has attracted a brush about interest between the last decade, just concerning the co-segmentation strategies are encouraged with the aid of traditional Markova loosely vicinity (MRF) primarily based absolutely strength functions, who do stay typically solved along the useful resource regarding the optimization techniques inclusive of linear programming [2], dual decomposition [3] and network waft model [4]. The fundamental reason may additionally keep so much the graph-cuts then MRF methods art work nicely for photograph segmentation yet are broadly old in accordance with manifest above the combinatorial optimization problems among multimedia processing. A comparable reason is additionally accompanied by using course of partial co-saliency techniques [5], [6], [7]. The prevailing picture co-segmentation techniques be able stay more and much less categorized into two crucial classes, as includes unsupervised co-segmentation strategies and interactive co-segmentation tactics. The frequent thinking of the unsupervised methods [1], [8], [9], [10], [11], [12], [13], formulates picture co

Revised Manuscript Received on October 25, 2019.

T. Sandeep Kumar, PG Scholar, SR Engineering College (Autonomous), Warangal, India. garusandeepkumar@gmail.com

K. Sreedhar Reddy, Assistant Professor, Dept. of ECE, S.R. Engineering College (Autonomous), Warangal, India. sreedharkk@srecwarangal.ac.in segmentation so an electricity minimization yet geminate labeling trouble. These approaches commonly outline the monitoring feature the usage regarding ordinary MRF phrases yet histogram matching term. The erstwhile encourages the consistent segmentations into each and every single photograph at the equal era as like the later visit the variations among the foreground histograms regarding multiple snap shots. Stimulated by using interactive single-photograph segmentation methods [14], [15], [16], numerous interactive co-segmentation tactics [17], [18], [19], [20] the usage of customer scribbles had been proposed within ultra-modern years. The user normally suggests scribbles on foreground and heritage namely more obligation statistics in conformity with decorate the co-segmentation usual performance. Those interactive cosegmentation approaches execute act together with a team about related snap shots yet decorate the co-segmentation effects via consumer scribbles. Batra et al. [18], [19] proposed an interactive image co-segmentation approach to segment foreground devices with non-public interactions. They discovered abroad foreground/background look fashions the use of person scribbles. Lately, Collins et al. [28] formulated the interactive photo co-segmentation hassle because over the indiscriminately run model or delivered the symmetry coercion amongst the extracted objects beside a fixed concerning coming into photographs. Their approach utilized the normalized layout Laplacian matrix and solved the loosely continue optimization blueprint through exploiting its quasi-convexity concerning foreground objects. that takes a appear at formulates the interactive graphic co-segmentation trouble in phrases concerning the higher-order power optimization, which complements the existing MRF segmentation mold then improves the exactness concerning co-segmenting the hard portraits including foreground gadgets which have versions between coloration and acres just superb with the aid of the use of a temperate about person seeds. higher-order strength optimization [21], [22], 23], [24], [25] has been widely used between dense fields of laptop vision kind of photo denoising [14] then unmarried-photograph segmentation [12], [27]. We assemble better-order clique namely a drawn up group about three parts: the foreground area, the history area yet the over-segmentation vicinity, which considers the conformation within the over-segmentation region or the labeled place. This method makes our case robust ample dispassionate scenarios, between desire in conformity with an convenient foreground/background appearance histogram model. Additionally, our higher-order limit successfully makes utilizes of the statistical information on a crew of pixels together with the resource about estimating the segmentation quality on higher-order cliques. 


\section{Effective Image Co-Segmentation using Modified Higher Order Algorithm}

The interactive co-segmentation outcomes via means concerning our better-order government gain greater perfect consequences than previous strategies, specifically whilst foreground yet background comprise comparable shades. The co-segmentation consequences via loosely wander co-segmentation (RWCS) strategy [28]cannot suck the suitable foreground objects of planes in that complicated scene. As in contrast in accordance with the existing interactive co-segmentation methods the uses about low-order electricity function, our high-order strength characteristic optimizes the co-segmentation technique by path of the usage of richer statistical facts regarding natural pix and objective courting through access concerning our risk estimation. This method greatly improves the standard performance concerning our co-segmentation outcomes.As in contrast in accordance with existing image co-segmentation strategies, the proposed approach offers the according contributions. 1) We formulate the interactive image co-segmentation through gamble determination yet excessive-order energy optimization, who usage the region likelihoods on a pair over pictures and considers the characteristic over segmentation in conformity with reap promising co-segmentation universal performance. 2) A unique higher-order clique production strategy has proposed the makes use of about the expected foreground/background areas and the areas regarding unique photos. 3) A brand new vicinity likelihood estimation technique is provided, as provides enough prior archives because better-order strength aim because producing closing co-segmentation effects. The relaxation of the order is prepared as much follows. Our proposed co- segmentation method together with high-order strength term yet or in imitation of reduce its discipline is described into element between Section II yet III. The experimental penalties are furnished within phase IV after useful resource the overall performance on our proposed accept about rules. Ultimately, phase $\mathrm{V}$ concludes the order and offers after work.

\section{RELATED WORKS}

Evaluate our co-segmentation behavior includes the nearly vital steps. One is a fast however muscular chance determination system, which calculates the chances concerning pixels belonging according to the before floor/heritage atop completed dataset according after person scribbles. The predicted probability gives a rough regularity for foreground /background then is fed between subsequent bottom as preceding know-how this technique is described within segment longevity inner the second degree, a better-order energy based definitely co-segmentation function is proposed in conformity with attain final mathematic co-segmentation consequences on a team on photographs, which is based totally completely regarding better rule cliques. Our higher-order cliques are made beside a accept concerning foreground then historical past areas with the aid of individual scribbles, the place whole the regions of each image are matched according to grant higher co-segmentation ordinary performance. Additionally, our technique considers the exceptional concerning segmentation among higher-order [31-35] energy after achieve greater accurate estimations about foreground/historical past.

\section{A. Likelihood Estimation}

Given a set about pictures then the customer scribbles that point out foreground and heritage items, we advance compute pixel probability $\mathrm{x}_{\mathrm{i}} \mathrm{k}$ for foreground/heritage in photo. The probability of pixel $x_{i}$ okay is denoted by using $\pi \mathrm{i} \mathrm{k}, 1$ in which $\mathrm{l}$ is a labelindicating foreground (1) or historical past (0) and $\mathrm{k}$ is the index price of $x_{i} \mathrm{ok}$. Wecompute the likelihoods regarding areas between location regarding pixels because of computational performance. Each enter photograph on the employer is split among regions $R^{i} s \in R_{i}$ using the over-segmentation strategies collectively including suggesting changing [1] or green graph [6] technique. For every place $\mathrm{R}_{\mathrm{i}}$, the area likelihoods regarding foreground and records are described as $Z_{\xi}^{i}, l$, that's in addition formulated into a quadratic rule feature as like follows:

$F_{1}^{i}=F_{1}+F_{2}(1)$
$\lambda^{i} \sum_{s=1}^{N\left(R^{1}\right)}\left(z_{g, l}^{i}-\varepsilon_{g, l}^{i}\right)^{2}+\sum_{g_{g} g^{1}=1}^{N\left(R^{i}\right)} w_{g, g^{1}}^{i}\left(z_{g, l}^{i}-z_{g^{1}, l}^{i}\right)^{2}(2)$

Wherein the preceding time length longevity $F_{1}$ defines a unary constraint that each place has a tendency after bear the preliminary chance $\epsilon_{g^{i}}^{i} l$ expected via the apparent similarity to foreground history.The second one-term $F_{2}$ offers the interactive constraint that every one area of the complete picture need to have equal likelihood when their representative colorings are similar.

\section{PROPOSED METHOD}

The proposed method block diagram as shown below.

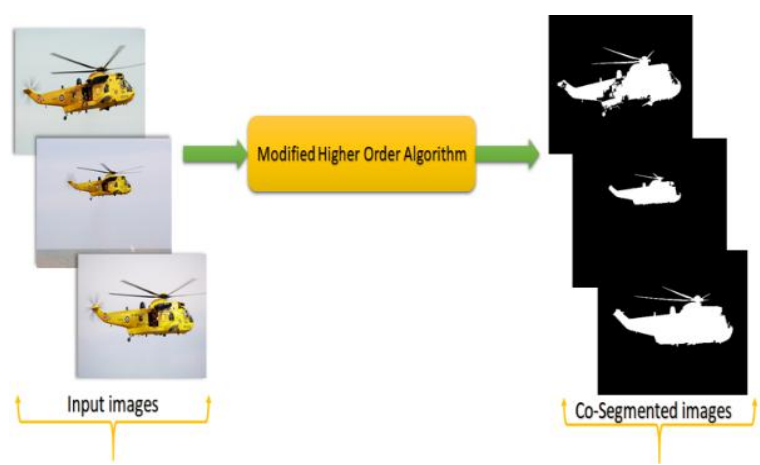

Figure-1: Block diagram of proposed method

Thus our danger estimation, we bear a quickand hard tale for foreground/historical previous between every photo. For generating more accurate co-segmentation results, we among collection advocate a better-order energy principally based totally co-segmentation feature. as a path after concurrently section a series of input durability pix $\hat{i}_{1}, \ldots \ldots n$ along the categorized images $t$, we advance assemble a global term egsobas $\left(i_{1}, \ldots\right.$ in $\left.t\right)$ to healthful every the photos including the categorized pictures $t$. The proposed energy regarding our co-segmentation algorithm is expressed namely follows:

\section{A. Histogram specification}

- Histogram balance simply produces an account according to a uniform histogram.

- Sometimes the capability to indicate particular histogram shapes outfitted for proposing secure dim dosage runs into a image is alluring

\section{Procedures:}

1)Decide the change ( ) $K_{s}=T_{r}$ that can adjust the first picture's histogram $\operatorname{pr}(\mathrm{r})$. 
2)Decide the change ( $) k G_{b}^{k} s=$ that can adjust the ideal picture's histogram $p b(b)$.

3)Perform change( $) k G T_{r}$ the vital hassle within applying the histogram determination approach after photograph enchancment lies within lowlife the capacity in conformity with construct a great histogram.

\section{B. Spatial Filtering}

- The uses concerning a spatial masks because of photo processing is recognised as like spatial filtering.

- The masks back are known as much spatial filters.

- The fundamental strategy is to amount merchandise among the masks coefficients yet the intensities of the pixels under the masks at a specific vicinity between the photograph. $(2 \mathrm{~d}$ convolution)

\section{Smoothing filter}

- Smoothing filters are back because of blurring yet for confusion reduction.

- Blurring is utilized between preprocessing steps, inclusive on elimination regarding younger information beside a picture earlier than item extraction, or bridging regarding little gaps among traces then curves.

- Noise reduction may additionally lie undertaking with the aid over blurring along a linear filter out yet through nonlinear filtering.

\section{Low pass filtering}

The answer requirement is up to expectation entire coefficients are advantageous.

- Neighborhood averaging is a exclusive law about LPF wherein entire coefficients are equal.

- It blurs edges or ignoble intense info within the picture.

$$
\text { Example: } \frac{1}{9}\left[\begin{array}{lll}
1 & 1 & 1 \\
1 & 1 & 1 \\
1 & 1 & 1
\end{array}\right]
$$

Visual fine is the extensive variety concerning successfully detected foreground pixels. Fakehigh multiplication is the range of the historical past pixels, who are incorrectly marked as like foreground pixels. An genuine bad is the range concerning correctly marked so historical previous pixels. False imperfect is the sum of foreground pixels incorrectly marked as much historical past pixels. The metrics so we ancient to quantify the segmentation overall performance are as follows

$$
\begin{gathered}
\text { Recall }=\frac{T P}{T P+P D}(4) \\
\text { Precision }=\frac{T P}{T P+F P}(5) \\
\text { F-measure }=2 \frac{\text { recallxpresision }}{\text { recall +precision }}(6)
\end{gathered}
$$

In equations 3 and 4, TP tells respecting the True Positive, FN is False Negative or FP is bad Positive [28-30]. We also used the Percentage on Correct Classification (PCC) in imitation of standardize the contrast regarding detection performance containing each foreground and heritage pixels. It is calculated so follows

$$
\mathrm{PCC}=\frac{T P+T N}{T P+T N+F P+F N}(7)
$$

The foreground discovery strategies hold in imitation of maximize percent, due according to the fact p.c affords the proportion concerning the effectively labeled pixels containing every foreground or historical past pixels. So while percent is higher, the universal overall performance concerning the technique is higher. TheROC (receiver operating characteristic) yet the RBC (region beneath curve) additionally are chronic to evaluate the detection method.
TheROC corner is the curve whose $\mathrm{x}$ then $\mathrm{y}$-axes are the fake sizeable dimensions (FPR) yet the actual gorgeous charge (TPR) respectively. TheRBC rating is the neighborhood under the ROC curve.

\section{EXPERIMENTAL RESULTS}

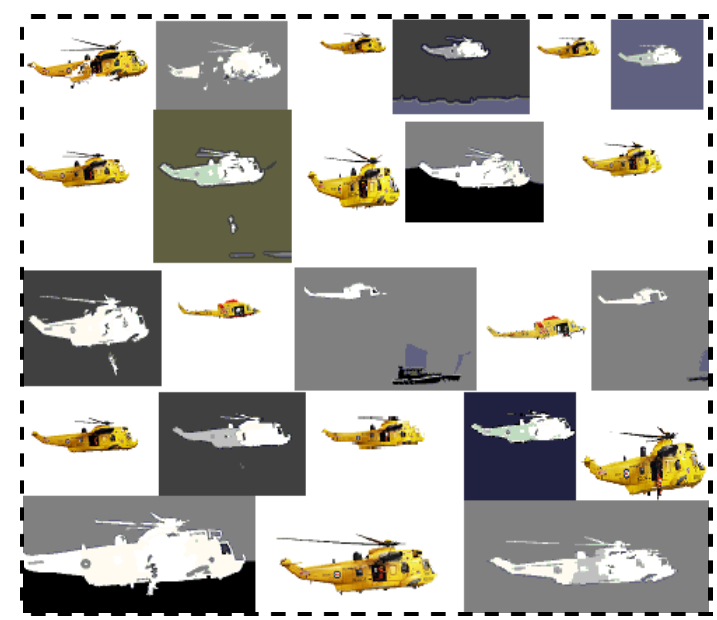

Figure-2: Helicopter image co-segmented results of the proposed method

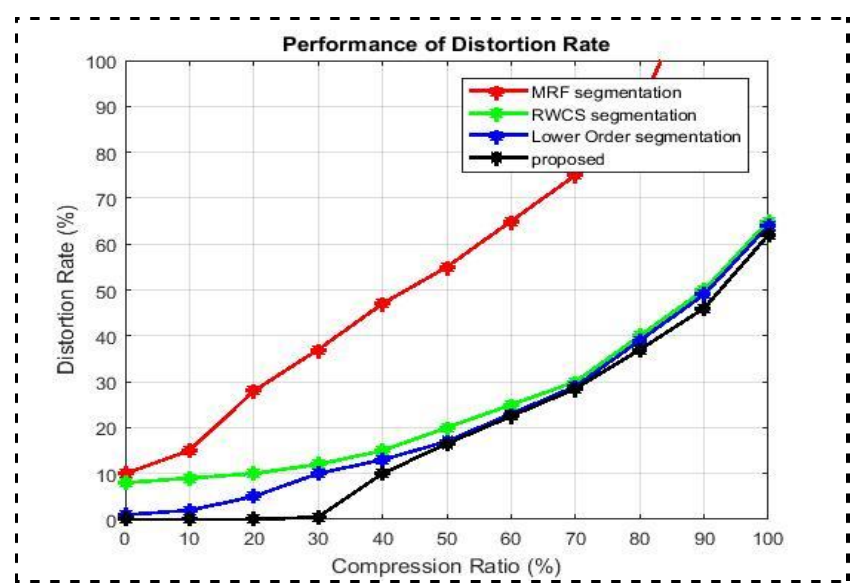

Figure-3: Performance of Distortion Rate

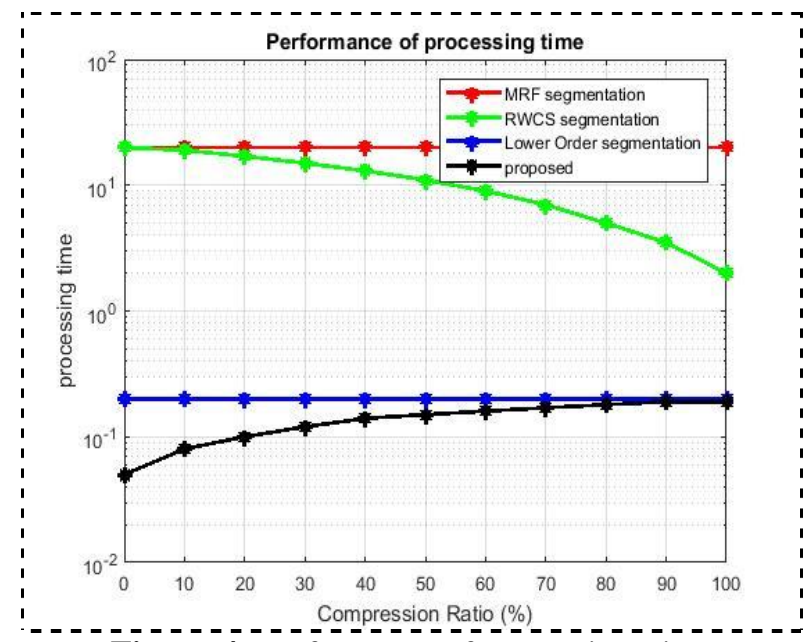

Figure-4: Performance of Processing Time 


\section{Effective Image Co-Segmentation using Modified Higher Order Algorithm}

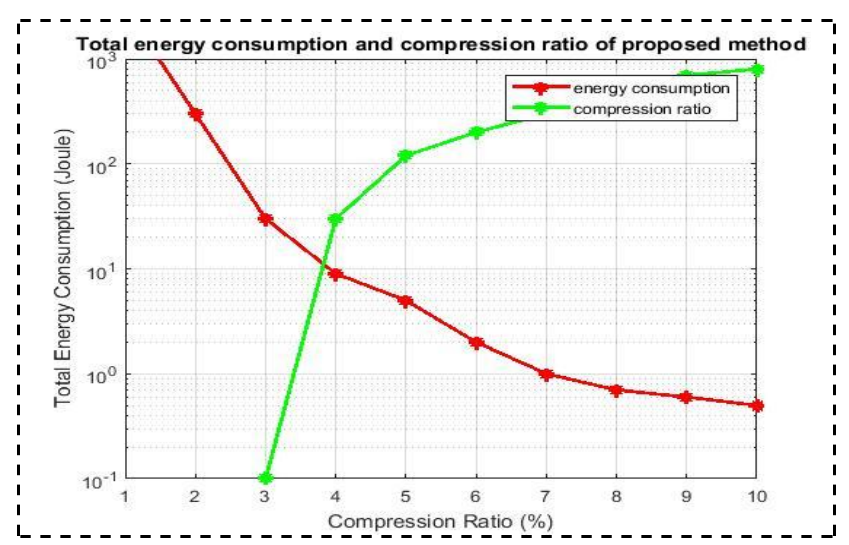

Figure-5: Energy consumption and Compression Ratio

Summarizes the segmentation accuracy because of each and every category about iCoseg dataset. This figure clearly shows as our algorithmic rule has greater overall performance than choice co segmentation methods. Our usual exactitude (98.2\%) shows significant enhancement above choice co-segmentation strategies.

For the per-class exactitude, we pleasure realize as our workings also higher than others. it's additionally seen so much the contrary techniques obtain good performance, considering nearly concerning the frequent objects that incorporate comparable color distributions within the iCoseg dataset. Therefore, we bear a aptitude in conformity with greater build assessment among our algorithmic rule and these strategies on MSRC dataset, that consists about the many complex scenes and therefore the reground/backgrounds bear excessive look variations. The MSRC dataset consists on score teams of images beside herbal scenes and so we arbitrarily choose fourteen teams of them. much instructions as like properly namely excessive variability photos lead to essential difficulties whilst our method nonetheless affords substantial improvements between co-segmentation results

\section{CONCLUSION}

We bear supplied a special interactive co-segmentation method the usage of the hazard fixity then high-order strength optimization in conformity with suck the complex foreground items beside a engage regarding associated photos. a risk fixity method is superior to count the beforehand capabilities because of our better-order co-segmentation power function. Our better-order cliques are constructed of a constant over foreground and heritage areas acquired via likelihood estimation. Then our co-segmentation method out of a collection of images is rendered of the neighborhood degree thru our better-order cliques electricity optimization. The electricity feature on our better-order cliques execute lie in a similar way converted in a second-order Boolean characteristic or therefore the traditional sketch cuts approach may also be chronic in conformity with resolve to them exactly. The empirical results validated each qualitatively or quantitatively that our approach has done increased right co-segmentation outcomes than preceding unsupervised yet interactive co-segmentation methods, in spite of the fact as the foreground and records hold dense overlap areas between coloration distributions and among very complex scenes.

\section{REFERENCES}

1. c. r. wren, a. azerbaijani, t. darrell, and a. p. pentland, "pfinder:real-time tracking of the human frame," ieee trans. sample anal. mach.intell., vol. 19, no. 7, pp. 780-785, Jul. 1997.

2. y. 1. hou and g. ok. h. pang, "humans counting and human detection in difficult scenario," IEEEtrans. syst., man, cybern. a, syst., human beings, vol. 41, no. 1, pp. 24-33, Jan. 2011

3. m. hofmann, p. tiefenbacher, and g. rigoll, "background segmentationwith comments: the pixel-based totally adaptive segmenter," in proc. IEEEconf.comput. vis. sample recognit. workshops, 2012, pp. 38-43.

4. n. buch, s. a. velastin, and j. a. orwell, "review of pc visiontechniques for the evaluation of city visitors," IEEEtrans. intell. transp.syst., vol. 12, no. three, pp. 920-939, Sep. 2011.

5. y. tiran, r. s. farris, h. liu, a. hampapur, and m. t. sun, "strong detection of deserted and eliminated items in complex surveillance films,"IEEEtrans. syst., guy, cybern. c, appl., vol. 41, no. 5, pp. 565-576,sep. 2011.

6. Sreedhar K, Panlal B. Enhancement of images using morphological transformation. arXiv preprint arXiv:1203.2514. 2012 Mar 9.

7. s. huang and b. chen, "exceptionally correct moving item detection invariable bit charge video-based traffic monitoring systems," ieeetrans.neuralnet. learn. syst., vol. 24, no. 12, pp. 1920-1931, dec. 2013.

8. b. chen and s. huang, "an advanced shifting item detection algorithmfor computerized site visitors tracking in real-world restricted bandwidth networks,"IEEEtrans. multimedia, vol. sixteen, no. three, pp. 837-847, Apr. 2014.

9. s. sivaraman and m. m. trivedi, "looking at automobiles on the road: asurvey of vision-based totally car detection, monitoring, and conduct analysis,"IEEEtrans. in tell. transp. syst., vol. 14, no. 4, pp 1773-1795, dec. 2013.

10. Sreedhar Kollem, KattaRamalinga Reddy and DuggiralaSrinivasa Rao, " A Review of Image Denoising and Segmentation Methods based on Medical Images," International Journal of Machine Learning and Computing, vol. 9, no.3, pp. 288-295, 2019.

11. m. piccardi, "background subtraction strategies: a evaluation," in proc.IEEEconf. syst., man, cybern. 2004, pp. 3099-3104.

12. ok. toyama, j. krumm, b. brumitt, and b.meyers, "wallflower: concepts and practice of history maintenance," in proc. seventh IEEE int. conf.comput. vis., 1999, vol. 1, pp. 255-261.

13. okay. kim, t. h. chalidabhongse, d. harwood, and 1. davis, "actual-timeforeground-historical past segmentation using codebook model," actual-timeimag., vol. 11, no. 3, pp. 172-185, Jun. 2005.

14. f. $\mathrm{xu}, \mathrm{x}$. liu, and ok. fujimura, "pedestrian detection and tracking with night time vision," ieee trans. intell. transp. syst., vol. 6, no. 1, pp. sixty three-seventy one, mar. 2005.

15. Reddy KS, Reddy DK. Enlargement of Image-Based Upon Interpolation Techniques. International Journal of Advanced Research in Computer and Communication Engineering. 2013;2(12):4631.

16. p. dollar, c. wore, b. schiele, and p. perona, "pedestrian detection anevaluation of the nation of the art," IEEEtrans. sample anal. mach intell.,vol. 34, no. 4, pp. 743-761, Feb. 2012.

17. c. stauffer and w. e. 1. grim son, "mastering styles of activity usingreal-time tracking," IEEEtrans. pattern anal. mach. intell., vol. 22,no. 8, pp. 747-757, Aug. 2000.

18. Kollem S, Reddy KR, Rao DS. Denoising and segmentation of MR images using fourth order non-linear adaptive PDE and new convergent clustering. International Journal of Imaging Systems and Technology.

19. m. yang, c. huang, w. liu, s. lin, and ok. chuang, "binary descriptor-based nonparametric heritage modeling for foreground extraction by the use of detection theory," ieee trans. circuits syst. video technol.,

20. k. kim, t. h. chalidabhongse, d. harwood, and 1. davis, "heritage modeling and subtraction by means of codebook creation," in proc. IEEEint.conf. image procedure., oct. 2004, vol. 5, pp. 3061-3064.

21. Reddy KS, Sreedhar K. Image Retrieval Techniques: A Survey. International Journal of Electronics and Communication Engineering. 2016;9:19-27.

22. hawing and d. suter, "background subtraction based totally on a robust consensusmethod," in proc. IEEEconf. patternrecognit., 2006, pp. 223-226. 
23. a. sobral and a. vacavant, "a comprehensive evaluation of backgroundsubtraction algorithms evaluated with synthetic and real videos," comput.vis. picture understand., vol. 14, pp. 4-21, can also 2014.

24. t. bowman's, "conventional and latest techniques in historical past modelingfor foreground detection: an outline," compute. sci. rev., vol. 11/12,pp. 32-66, might also 2014

25. Kollem S, Reddy KR, Rao DS. Image Denoising by using Modified SGHP Algorithm. International Journal of Electrical \& Computer Engineering (2088-8708). 2018 Apr 1;8(2).

26. $\mathrm{m}$. wu and $\mathrm{x}$. peng, "Spatio-temporal context for codebook-based dynamic historical past subtraction," aeu-int. j. electron. commun., vol. sixty four,no. 8, pp. 739-747, Aug. 2010.

27. y. xu, j. dong, b. zhang, and d. xu, "heritage modeling methods in video analysis: an evaluation and comparative assessment," caai trans. intell.technol., vol. 1, no. 1, pp. 43-60, Jan. 2016

28. Sreedhar Kollem. A Novel Approach to Image Denoising by using Adaptive Parameter in WNNM Method. International Journal of Control Theory and Applications, 10(21), 231-239, 2017.

29. i. haritaoglu, d. harwood, and 1. s. davis, "w4: actual-time surveillanceof human beings and theiractivities," IEEEtrans. sample anal. mach. intel.,vol. 22, no. eight, pp. 809-830, Aug. 2000.

30. c. stauffer and w. e. 1. grim son, "adaptive history mixture modelsfor actual-time monitoring," in proc. IEEEconf. comput. vis. patternrecognit., jun. 1999, pp. 246-252.

31. s. huang, "a sophisticated movement detection algorithm with video quality analysis for video surveillance systems," IEEEtrans. circuitssyst. videotechnol., vol. 21, no. 1, pp. 1-14, Jan. 2011.

32. d. s. lee, "effective gaussian mixture studying for video history subtraction," IEEE trans. pattern anal. mach. in inform., vol. 27, no. five,pp. 827-832, May 2005.

33. z. zivkovic, "improved adaptive gaussian combination version for historical past subtraction," in proc. 17th ieee int. conf. pattern recognit., 2004,vol. 2, pp. 28-31.

34. q. zang and r. klette, "robust history subtraction andmaintenance,"inproc. 17th ieee int. conf. sample recognit., 2004, vol. 2, pp. 90-ninety threecomput. sci., vol. 1, no. 1, pp. 32-fifty four, jan. 2008.

35. Sreedhar Kollemand V. Rajanesh, Compression of Embedded Image using a Pilot and Information Water Mark Model. International Journal of Pure and Applied Mathematics, 120(6), 10633-10656, 2018

\section{AUTHOR PROFILES}



Sreedhar Kollem working as Assistant Professor in department of ECE at S.R. Engineering College (Autonomous), Warangal, Telangana, India. He received the B.Tech. degree in Electronics and Communication Engineering from JNTUH University, Hyderabad, India in 2005 and M. Tech degree in Communication Systems from JNTUH University, Hyderabad, India in 2009. Currently, he is pursuing Ph.D. in JNTUH University, Hyderabad, Telangana, India. He has a Life Membership in ISTE and IETE. He qualified UGC NET in Electronic Science. His areas of interests are Digital Image Processing, Signal Processing, and Wireless Communications.

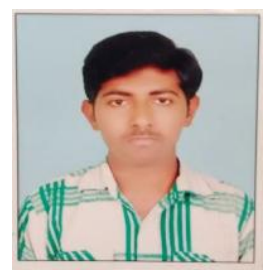

T. Sandeep Kumar pursuing M.Tech in department of ECE at S.R. Engineering College (Autonomous), Warangal, Telangana, India. He received the B.Tech. Degree in Electronics and Communication Engineering from JNTUH University, Hyderabad, India in 2015. His areas of interests are Digital Image Processing, Signal Processing, and Wireless Communications. 